\title{
CDK5RAP3, a New BRCA2 Partner That Regulates DNA Repair, Is Associated with Breast Cancer Survival
}

\author{
Jordi Minguillón 1,2,*迆, María José Ramírez ${ }^{1,2} \mathbb{D}$, Llorenç Rovirosa ${ }^{1,2} \mathbb{D}$, Pilar Bustamante-Madrid ${ }^{1,2}$, \\ Cristina Camps-Fajol 1,2, Gorka Ruiz de Garibay ${ }^{3} \mathbb{D}$, Hermela Shimelis ${ }^{4,5}$, Helena Montanuy ${ }^{1,2}$, Roser Pujol 1,2, \\ Gonzalo Hernandez 1,2 (D), Massimo Bogliolo ${ }^{1,2}$, Pau Castillo ${ }^{1,2}$, Penny Soucy ${ }^{6}$, Griselda Martrat ${ }^{3}$, \\ Antonio Gómez ${ }^{7} \mathbb{D}$, Daniel Cuadras ${ }^{3} \mathbb{D}$, María J. García ${ }^{8,+}$, Javier Gayarre ${ }^{8}$, CIMBA , Conxi Lázaro $^{9}$, \\ Javier Benítez $^{8}$, Fergus J. Couch ${ }^{4,5}$, Miquel Angel Pujana ${ }^{3}$ (D) and Jordi Surrallés ${ }^{1,2, *(\mathbb{D})}$
}

check for updates

Citation: Minguillón, J.; Ramírez, M.J.; Rovirosa, L.;

Bustamante-Madrid, P.; Camps-Fajol,

C.; Ruiz de Garibay, G.; Shimelis, H.;

Montanuy, H.; Pujol, R.; Hernandez,

G.; et al. CDK5RAP3, a New BRCA2

Partner That Regulates DNA Repair,

Is Associated with Breast Cancer

Survival. Cancers 2022, 14, 353.

https://doi.org/10.3390/

cancers14020353

Academic Editor: Obul

Reddy Bandapalli

Received: 27 September 2021

Accepted: 30 October 2021

Published: 12 January 2022

Publisher's Note: MDPI stays neutral with regard to jurisdictional claims in published maps and institutional affiliations.

Copyright: (c) 2022 by the authors. Licensee MDPI, Basel, Switzerland. This article is an open access article distributed under the terms and conditions of the Creative Commons Attribution (CC BY) license (https:/ / creativecommons.org/licenses/by/ $4.0 /)$.
1 Genomic Instability Syndromes and DNA Repair Group and Join Research Unit on Genomic Medicine UAB-Sant Pau Biomedical Research Institute, Hospital de la Santa Creu i Sant Pau, 08041 Barcelona, Spain; MariaJose.Ramirez@uab.cat (M.J.R.); lrovirosa@carrerasresearch.org (L.R.); pbustamante@iib.uam.es (P.B.-M.); CCampsF@santpau.cat (C.C.-F.); hmontanuy@vhio.net (H.M.); MariaRoser.Pujol@uab.cat (R.P.); ghernandezv@uic.es (G.H.); massimo.bogliolo@uab.es (M.B.); pau.castillo@uab.cat (P.C.)

2 Center for Biomedical Network Research on Rare Diseases (CIBERER) U-745, 08041 Barcelona, Spain

3 Program against Cancer Therapeutic Resistance (ProCURE), Breast Cancer and Systems Biology, Catalan Institute of Oncology (ICO), Bellvitge Institute for Biomedical Research (IDIBELL), L'Hospitalet del Llobregat, 08908 Barcelona, Spain; gderuiz@cnio.es (G.R.d.G.); griseldamartrat@gmail.com (G.M.); daniel.cuadras@sjd.es (D.C.); mapujana@iconcologia.net (M.A.P.)

4 Department of Laboratory Medicine and Pathology, Mayo Clinic, Rochester, MN 55901, USA; hshimelis@geisinger.edu (H.S.); couch.fergus@mayo.edu (F.J.C.)

5 Department of Health Sciences Research, Mayo Clinic, Rochester, MN 55901, USA

6 Genomics Center, Centre Hospitalier Universitaire de Québec Research Center and Laval University, Quebec City, QC G1E 6W2, Canada; penny.soucy@crchul.ulaval.ca

7 Cancer Epigenetics and Biology Program, Bellvitge Institute for Biomedical Research (IDIBELL), L'Hospitalet del Llobregat, 08908 Barcelona, Spain; antonio.gomez@vhir.org

8 Human Cancer Genetics Program, Spanish National Cancer Research Centre (CNIO), 28029 Madrid, Spain; mjgarcia@cnio.es (M.J.G.); jnavarro507@hotmail.com (J.G.); javier.benitez@cnio.es (J.B.)

9 Hereditary Cancer Program, Catalan Institute of Oncology (ICO), Bellvitge Institute for Biomedical Research (IDIBELL), L'Hospitalet del Llobregat, 08908 Barcelona, Spain; clazaro@iconcologia.net

* Correspondence: jordi.minguillon@uab.cat (J.M.); jordi.surralles@uab.cat (J.S.)

+ Current address: Computational Oncology Group, Structural Biology Program, Spanish National Cancer Research Centre (CNIO), 28029 Madrid, Spain.

$\ddagger$ Consortium of Investigators of Modifiers of BRCA1/2. Department of Public Health and Primary Care, Centre for Cancer Genetic Epidemiology, University of Cambridge, Cambridge CB1 8RN, UK.

Simple Summary: $B R C A 2$ is an essential gene for DNA repair by homologous recombination and is often mutated in families at risk of breast and ovarian cancer. In this study we identified CDK5RAP3 tumor suppressor as a new BRCA2-interacting protein. CDK5RAP3 negatively regulates DNA repair of double-strand breaks, providing a new mechanism of DNA damage resistance. Consistently, gene expression data analysis showed CDK5RAP3 overexpression in breast cancer is associated with poorer survival. Finally, we identified common genetic variations in the CDK5RAP3 locus as potentially associated with breast and ovarian cancer risk in a large cohort of $B R C A 1$ and $B R C A 2$ mutation carriers. 
patients. Finally, we identified common genetic variations in the CDK5RAP3 locus as potentially associated with breast and ovarian cancer risk in $B R C A 1$ and $B R C A 2$ mutation carriers. Our results uncover CDK5RAP3 as a critical player in DNA repair and breast cancer outcomes.

Keywords: BRCA2; breast cancer; CDK5RAP3; DNA repair; chemoresistance

\section{Introduction}

BRCA2, the breast cancer type 2 susceptibility gene product, is a master regulator of the DNA damage response pathway. BRCA2 is involved in DNA repair by homologous recombination (HR) and maintains genomic stability to counteract the mutational load due to double-strand breaks (DSBs) and interstrand crosslink (ICL) inducers [1,2]. BRCA2 is a 3418-amino-acid protein with multiple domains that interact with numerous functionally related proteins, such as RAD51, MDC1 and FANCD2 [3-5]. Monoallelic and biallelic mutations in BRCA2 cause hereditary breast and ovarian cancer and Fanconi anaemia (FA), respectively [6-9]. Similar causative associations have been described for other genes of the FA/BRCA pathway, including BRCA1, PALB2, and RAD51C [10-15].

CDK5 regulatory subunit-associated protein 3 (CDK5RAP3, also known as C53, LZAP or IC53) is a tumor suppressor that activates p53, induces genotoxic-dependent apoptosis, inhibits the G2/M checkpoint via CHK1 and CDK1, and reduces NF- $\mathrm{KB}$ activity, cellular invasion, and tumor growth [16-19]. Low levels of CDK5RAP3 are associated with a subset of head and neck squamous cell carcinomas (HNSCCs) and hepatocellular carcinomas (HCCs) as well as with poor survival $[18,20]$. In this study, a screening for physical interactors of BRCA2 identified CDK5RAP3, which emerged as a critical regulator of DNA DSB repair that is associated with breast cancer outcomes.

\section{Materials and Methods}

\subsection{Reagents and Antibodies}

Mitomycin C (MMC, M0503), diepoxybutane (DEB, ref. 202533-16) 5-bromo-2deoxyuridine (BrdU, B5002) and Hoechst 33258 (B2883) and IGEPAL (I8896) were from Sigma (Madrid, Spain). Ethidium monoazide bromide (EMA, e1374) and Sytox Green (S7020) were from Thermofischer Scientific (Barcelona, Spain). PARP inhibitor Olaparib (S1060) was from Selleckchem (Munich, Germany). Propidium iodide (P3566) and RNAse (12091021) were from Invitrogen (Barcelona, Spain). Colcemid was from Gibco (Barcelona, Spain). Antibodies used included: mouse monoclonal to CDK5RAP3 (ab57817), rabbit polyclonal to BRCA2 (ab123491), mouse monoclonal to RPA32 (ab2175), rabbit polyclonal to actin (ab1801), rabbit polyclonal to GAPDH (ab9485) and mouse monoclonal to vinculin (ab18058) were from Abcam (Cambridge, UK). Rabbit polyclonal to RAD51 (SC8349) was from Santa Cruz biotechnology (Heidelberg, Germany).

\subsection{Cell Lines and Plasmids}

U2OS, HeLa, and stable cell lines U2OS-CherryCDK5RAP3, U2OS DR-GFP, U2OS SA-GFP, U2OS EJ5-GFP and HeLa SA-GFP were grown in DMEM (Biowest, cat. L0104, (Nuaillé, France) supplemented with 10\% FBS (Biowest, cat. S181B) and plasmocin $0.1 \mathrm{mg} / \mathrm{L}$ (Invivogen, cod ant-mpt, Toulouse, France). I-SCEI-expressing plasmid pCBAS, empty vector (pCAGGS) and GFP-expressing plasmid NZE-GFP was kindly provided by Dr. Maria Jasin (Memorial Sloan Kettering Cancer Center, New York). SA-GFP (plasmid 41594) and EJ5-GFP (plasmid 44026) were from Addgene (Watertown, MA, USA. For pmCherryCDK5RAP3 construct, CDK5RAP3 cDNA was amplified with primers flanking the Sal I restriction site and cloned into pmCherry-C1 vector (Clontech, Saint-Germain-en-Laye, France). For DNA and siRNA transfection, we used lipofectamine 2000 (cat. 11668) and lipofectamine RNAiMax (cat. 13778), respectively, from Invitrogen. 


\subsection{Western Blot}

U2OS, HeLa or HEK cells were seeded on 6 well plates, $24 \mathrm{~h}$ later were treated with hydroxyurea, MMC or left untreated, and $24 \mathrm{~h}$ later cells were lyzed and SDS-PAGE and blotting with indicated antibodies performed as previously described [21]. The uncropped western blots are shown in Figure S5.

\section{4. siRNA Transfection Oligonucleotides}

Oligonucleotides were purchased from Sigma with the following sequences: Luciferase 5'-CGU ACG CGG AAU ACU UCG A-3'; CDK5RAP3-1 5'-GGC AGG AGA UUA UAG CUC U-3'; CDK5RAP3-2 5'-GGU UCG GAA UGU CAA CUA U-3'; CDK5RAP3-3 5'-CCC UGA CAC UGC UUG AAU A-3'; BRCA2 5'-GGA UUA UAC AUA UUU CGC A-3'.

\subsection{Analysis of BRCA2 Interactions}

Yeast two hybrid screens were carried out as previously described [22]. To confirm CDK5RAP3 interaction with BRCA2, HEK 293T cells expressing an empty vector or FlagBRCA2 were treated with double thymidine block $(10 \mathrm{mM})$ followed by a $9 \mathrm{~h}$ release to enrich S-phase cells, or left untreated. Cells were lysed and subjected to immunoprecipitation (IP) with anti-Flag M2 antibody. Immunoprecipitates and whole cell lysates were immunoblotted with CDK5RAP3, BRCA2 or actin antibodies. In other experiments, HEK 293T cells were left untreated or synchronized to S-phase by treatment with 2 mM hydroxyurea for $18 \mathrm{~h}$. Cells were lysed and subjected to IP with anti-IgG or anti-BRCA2 antibody, followed by immunoblotting with CDK5RAP3, BRCA2, geminin or actin antibodies.

\subsection{Laser Microirradiation Experiments}

Laser microirradiation experiments were reported earlier [23]. Briefly, pmCherry CDK5RAP3-transfected U2OS cells were sorted twice (FacsJazz, Becton Dickinson, Madrid, Spain) for stable transfection. Cells were plated, stimulated with $10 \mu \mathrm{M}$ BrdU for $24 \mathrm{~h}$, and before microscope analysis pre-treated $10 \mathrm{~min}$ at $37^{\circ} \mathrm{C}$ with $5 \mu \mathrm{g} / \mathrm{mL}$ Hoescht 33342 (Life Technologies, Barcelona, Spain). Images were taken with an Olympus Fluoview 1000 confocal microscope (Olympus, Barcelona, Spain). For laser microirradiation studies, cells were microirradiated with $5 \mathrm{~s}$ pulses of spots or lines with the $405 \mathrm{~nm}$ laser at full power and images were taken every $5 \mathrm{~s}$ for up to $5 \mathrm{~min}$ after localized DNA damage. For time course experiments, Cherry-CDK5RAP3 fluorescence was quantified from at least 30 cells and averaged.

\subsection{Survival and Clonogenic Assays}

Survival experiments were reported before [24]. Briefly, U2OS or HeLa cells were transfected twice with luciferase, BRCA2 or CDK5RAP3 siRNA at a final concentration of 20-40 nM in 6-multiwell plates. $24 \mathrm{~h}$ later, $1 \times 10^{5}$ cells were seeded in duplicate in 6-multiwell plates (or $35 \mathrm{~mm}$ dish plates for irradiation) and left untreated for $24 \mathrm{~h}$ more. Cultures were then exposed to mitomycin C (MMC), olaparib or $\gamma$-irradiated at the indicated doses. Three to four days after the treatment, cells were rinsed with PBS, harvested by trypsinization and counted. Survival is reported as the percentage relative to untreated controls. For clonogenic assays, cells were plated and treated with MMC or $\gamma$-irradiated at the indicated doses, left to grow for 10-14 days, and Giemsa stained; then, colonies were finally counted.

\subsection{In Vitro Flow Cytometric Micronuclei (FCM) Assay}

FCM assay was reported previously [21]. Briefly, 250,000 cells/well were plated in 6-well plates. Cells were then treated with ICL inducer DEB $(0.05$ or $0.1 \mu \mathrm{g} / \mathrm{mL})$ for 3 days. After treatment, trypsinized cells were centrifuged at $800 \mathrm{rpm}$ for $8 \mathrm{~min}$. Supernatant was removed and cells were resuspended by vortexing. $25 \mu \mathrm{L}$ of EMA solution $(0.125 \mathrm{mg} / \mathrm{mL}$ in PBS with $2 \%$ of FBS) was added to cell suspension. For the photo-activation step, plates were placed on ice under the visible light from a light bulb located $30 \mathrm{~cm}$ above cells for $20 \mathrm{~min}$. 
After the photo-activation step (EMA covalently binds the DNA of cells with disrupted membrane integrity - dead/dying cells), plates were protected from light exposure for the remaining steps of the staining procedure. $100 \mu \mathrm{L}$ of cold PBS with $2 \%$ FBS were added, and cells were centrifuged at $800 \mathrm{rpm}$ for $8 \mathrm{~min}$. Supernatant was discarded and cells were resuspended by vortexing. Cell suspension was kept in room temperature for $20 \mathrm{~min}$ before cell lysis. Cells were then lysed in a two-step procedure. $100 \mu \mathrm{L}$ of lysis solution 1 $(0.584 \mathrm{mg} / \mathrm{mL} \mathrm{NaCl}, 1 \mathrm{mg} / \mathrm{mL}$ sodium citrate, $0.3 \mu \mathrm{L} / \mathrm{mL}$ IGEPAL, $1 \mathrm{mg} / \mathrm{mL}$ RNase A, $0.2 \mu \mathrm{M}$ Sytox Green) were added. Samples were incubated at room temperature for $1 \mathrm{~h}$. After the incubation, $100 \mu \mathrm{L}$ of lysis solution $2(85.6 \mathrm{mg} / \mathrm{mL}$ sacarose, $15 \mathrm{mg} / \mathrm{mL}$ citric acid and $0.2 \mu \mathrm{M}$ Sytox Green) were added into the samples and immediately vortexed. Samples were incubated at room temperature for $30 \mathrm{~min}$ and were stored into a cold chamber overnight until samples were measured. Flow cytometry analysis was performed with a FACSCalibur (Becton Dickinson). At least 20,000 EMA negative-Sytox Green positive events (divided nuclei) were gated per sample. To exclude events that are not $\mathrm{MN}$ or nucleus from live cells, we used different gates for analysis that included forward scatter, side scatter, Sytox Green and EMA fluorescence (see Supplementary Figure S2A for details). Micronuclei $(\mathrm{MN})$ frequency was expressed as the number of MN per thousand nuclei obtained in the G plot. Percentage of cells arrested in the G2/M phase was obtained in the H plot.

\subsection{DNA Repair Assays}

HR, SSA and NHEJ assays were performed essentially as described [25-27]. For the HR assay, the U2OS DR-GFP stable cell line was used. For SSA and NHEJ assays, U2OS and HeLa cell lines were transiently transfected with SA-GFP or EJ5-GFP plasmids, selected with puromycin, and clonal cell lines obtained from limited dilution. Stable cell lines were confirmed to have specific GFP fluorescence upon I-SCEI but not upon empty vector transfection. For DNA repair assays, I-SCEI-endonuclease-expressing vector, empty vector or GFP-expressing vector were transfected in U2OS or HeLa cell lines for DNA repair assays. Cells were transfected with NZE-GFP, pCBAS or pCAGGS for 16-24 h with the indicated plasmids with Lipofectamine 2000 (Invitrogen) and, 24 h, later collected and analyzed by cell cytometry.

\subsection{Analysis of Publicly Available Cancer Transcriptomic Datasets}

To analyze the effect of CDK5RAP3 expression on the prognostics of ovarian and breast cancer patients, we generated Kaplan-Meier survival curves of these cancers with low or high expression of CDK5RAP3 by using Kaplan-Meier Plotter (www.kmplot.com accessed on 1 October 2017) [28]. For TCGA analysis, pre-processed and normalized gene expression data were downloaded from the corresponding TCGA repository (http: / / tcga-data.nci.nih.gov / tcga/tcgaHome2.jsp (accessed on 3 July 2021)). Expression profiles were clustered using the PAM50 signature. The signatures were compiled from the corresponding publications and corresponded to up-regulated resistance-associated genes. The CDK5RAP3 signature correlations were computed using the PCC and the average $Z$-score value per gene set. The profiles were clustered using the R software package cluster.

\section{Results}

\subsection{CDK5RAP3 Interacts with BRCA2 and Localizes to DNA Damage}

We screened for BRCA2 interactors by applying the yeast two-hybrid system and five baits based on Pfam-predicted domains and PONDR-predicted disordered regions [22], which overall covered most of the functionally important BRCA2 domains/regions (Figure 1A). The helical domain baits identified two potential interactors: CDK5RAP3 and LRRC45. The tower domain baits identified two additional potential interactors: SIPA1L1 and TACC3. Of these, we focused on CDK5RAP3, owing to its previously reported role as a tumor suppressor in hepatocarcinoma [18]. The CDK5RAP3-BRCA2 interaction was confirmed by coimmunoprecipitation (co-IP) in HEK293T cells. Cells were transfected with Flag-tagged BRCA2 and lysates were immunoprecipitated with anti-Flag 
antibody. Untransfected cells were immunoprecipitated with anti-BRCA2 antibody. As seen in Figure 1B,C, CDK5RAP3 interacted with BRCA2 in cells overexpressing Flag-BRCA2, as well as with endogenous BRCA2, only when cells were synchronized in the S-phase with double thymidine block or hydroxyurea treatment. These findings identified CDK5RAP3 as a novel physical interactor with BRCA2 and suggested that the association occurs during the S-phase of the cell cycle.
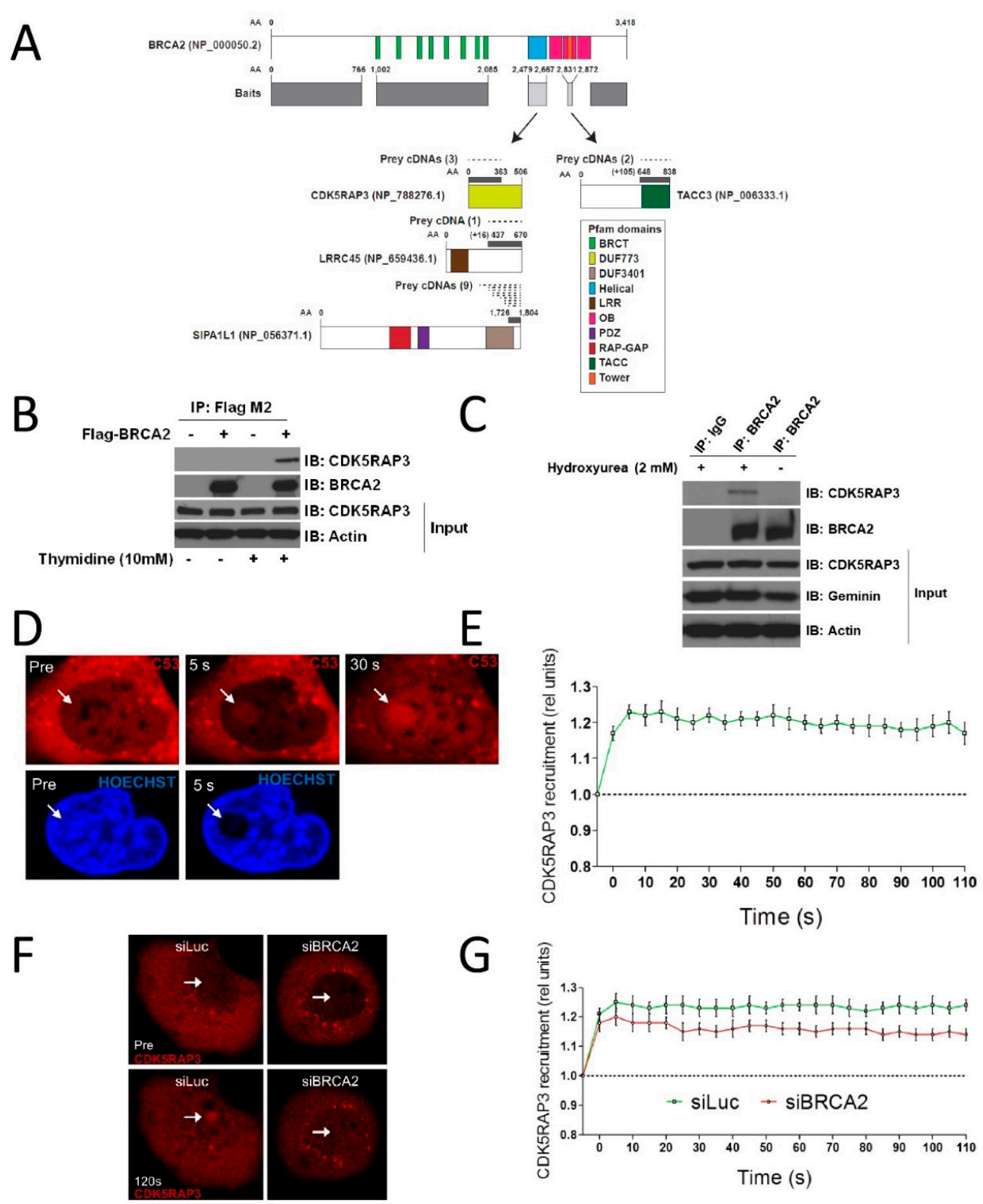

Figure 1. CDK5RAP3 interacts with BRCA2 and relocates to DNA damage. (A) BRCA2 fragments used as baits for $\mathrm{Y} 2 \mathrm{H}$ assay and potential interactors found (see materials and methods). (B) BRCA2 immunoprecipitation in HEK 293T cells expressing empty vector or Flag-BRCA2, double thymidine treated to enrich S-phase cells, or left untreated. (C) Endogenous BRCA2 IP in HEK 293T cells, S-phase synchronized with hydroxyurea or left untreated. (D) Laser micro-irradiation of U2OS cells stably expressing Cherry-CDK5RAP3. Representative image shows very fast CDK5RAP3 localization to DNA damage sites. (E) Time course of CDK5RAP3 DNA damage localization. (F) CDK5RAP3 localization after micro-irradiation in U2OS cells upon BRCA2 depletion. Representative image shows reduced CDK5RAP3 relocation in BRCA2 depleted cells. (G) Time course of CDK5RAP3 relocation to DNA damage upon BRCA2 depletion. 
Once the CDK5RAP3-BRCA2 interaction was confirmed, we checked whether CDK5RAP3 relocalized to the site of DSBs by laser microirradiation experiments and confocal microscopy. As seen in Figure 1D,E, CDK5RAP3 relocalized to DNA damage shortly after microirradiation, and nuclear distribution was also enhanced after DNA damage. Depletion of BRCA2 resulted in reduced relocation of CDK5RAP3 to DNA damage, suggesting that the role of CDK5RAP3 in DNA damage repair is functionally connected to BRCA2 (Figure 1F,G).

\subsection{CDK5RAP3-Depleted Cells Are Resistant DNA Damage}

It was previously reported that CDK5RAP3 affects sensitivity to DNA damage, as its inhibition reduced cell death and apoptosis in response to $20 \mu \mathrm{M}$ etoposide and $2.5 \mathrm{~Gy}$ radiation treatment in HeLa cells [16]. Accordingly, we examined the effect of CDK5RAP3 depletion in the context of the HR pathway. In particular, we compared the DNA damage sensitivity of CDK5RAP3-depleted U2OS cells with that of BRCA2-depleted cells. Small interfering RNAs (siRNAs) were used to deplete CDK5RAP3 or BRCA2, and the expression reduction was confirmed by Western blot (Figure 2A, right panel and Figure S5). We then performed survival and clonogenic assays after treating the cells with increasing doses of an ICL inducer (MMC), a DSB inducer (ionizing radiation, IR), or a PARP inhibitor (olaparib). As expected, inhibition of BRCA2 rendered the cells highly sensitive to DNA-damaging agents (Figure 2A-D). However, CDK5RAP3-depleted cells became resistant to MMC (Figure 2A,B), IR (Figure 2A,C), and to a lesser extent, olaparib (Figure 2D). Similar results were seen in HeLa cells (data not shown). Finally, to discard the potential interference of cell growth in mutagen sensitivity, we analyzed cell cycle progression and proliferation, which were similar in CDK5RAP3-depleted cells in comparison with control cells (data not shown).
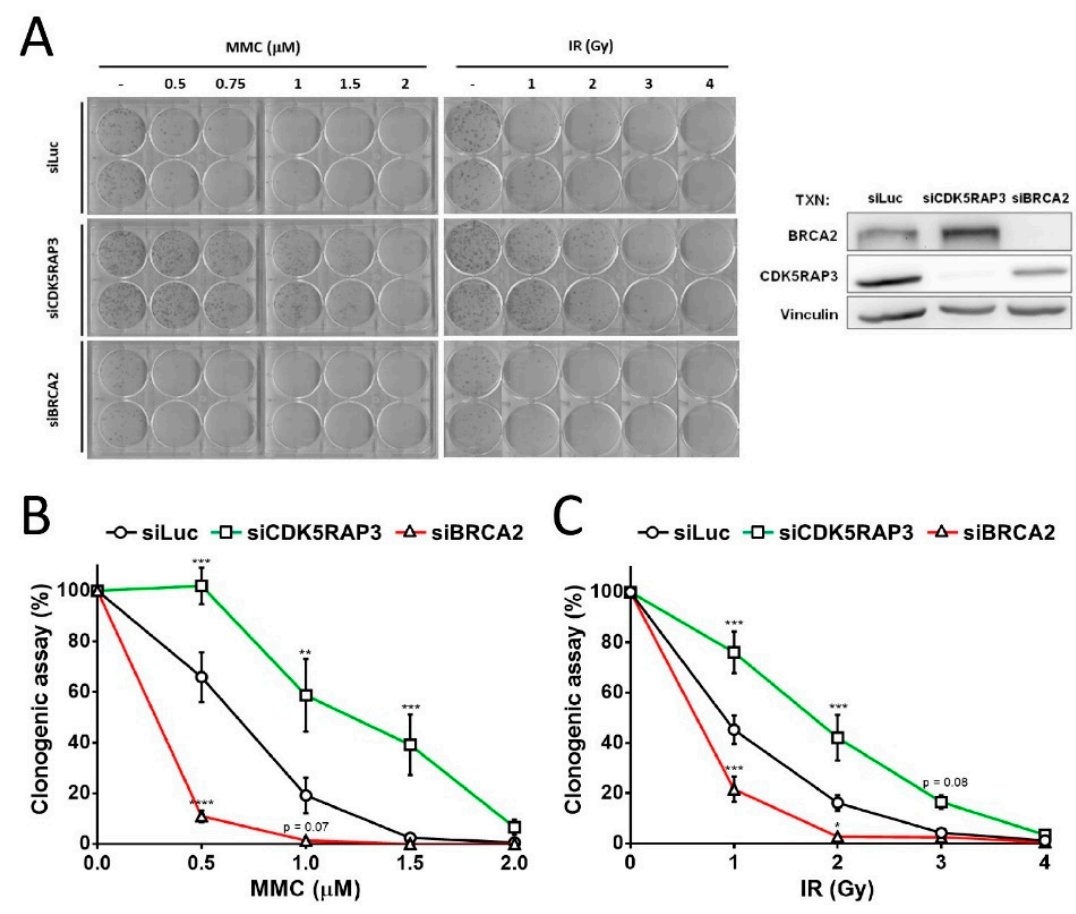

Figure 2. Cont. 


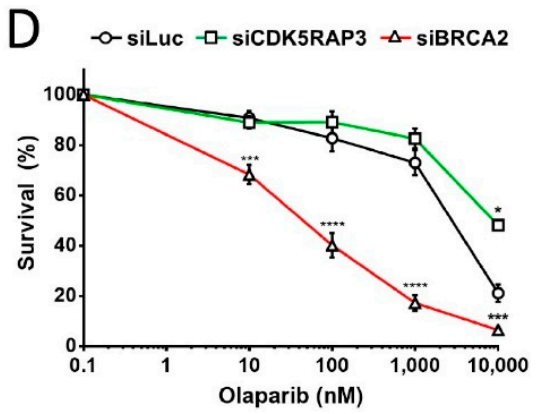

Figure 2. CDK5RAP3-depletion confers DNA damage resistance. (A) Clonogenic assay of U2OS cells transfected with siRNA targeting CDK5RAP3, BRCA2 or luciferase as a control (see materials and methods). Cells were plated and treated with different MMC (left) or ionizing radiation (right) doses for 10-14 days and Giemsa stained. Image shown is representative of at least three independent experiments with similar results. Right panel shows expression of siRNA targeted genes by Western blot. (B,C) Clonogenic assay graphs from averaged experiments done as in (A) in U2OS cells treated with MMC (B) or IR (C). (D) Survival assay of U2OS cells transfected as in (A), treated with different doses of PARP inhibitor olaparib. Graphs from $(B, C)$ show mean \pm SEM of at least 3 independent experiments. ${ }^{*} p<0.05,{ }^{* *} p<0.01,{ }^{* * *} p<0.001,{ }^{* * * *} p<0.0001$.

\subsection{CDK5RAP3 Regulates DSB Repair and Genomic Instability}

Because BRCA2-deficient cells are HR defective [1,2], we examined whether CDK5RAP3 similarly regulates DSB repair. An in vitro HR assay was performed in U2OS cells by detecting the HR-dependent correction of an incomplete GFP expression cassette inserted in the genome (Figure 3A left panel) in the presence or absence of CDK5RAP3 [25]. Control cells with intact HR exhibited efficient functional GFP expression (Figure 3B, upper left panel). As expected, BRCA2 inhibition strongly abolished HR repair (Figure 3B upper right and Figure 3C). Interestingly, CDK5RAP3 downregulation resulted in a marked increase in the percentage of fluorescent cells (Figure 3B, upper middle and Figure 3C).

A
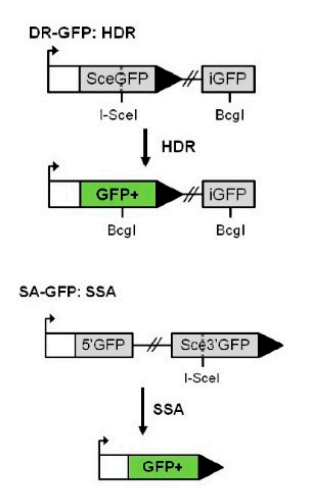

C

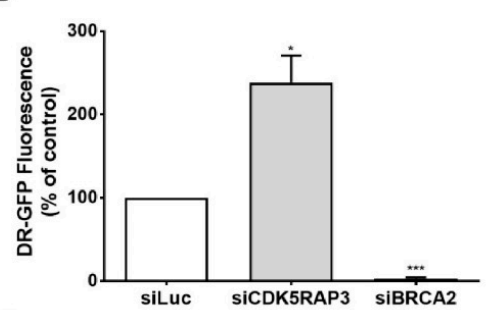

B
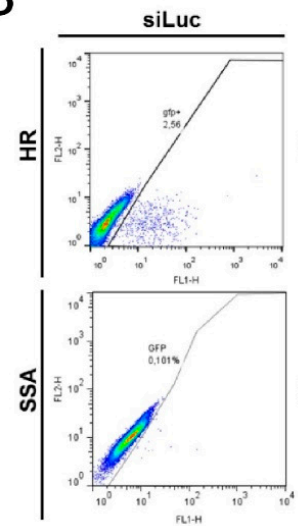

D
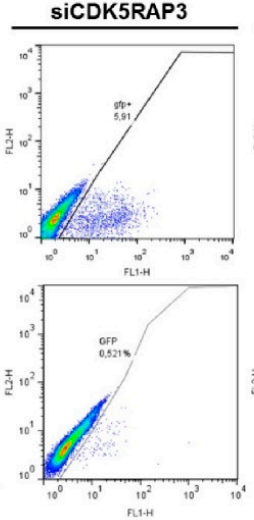

(1)

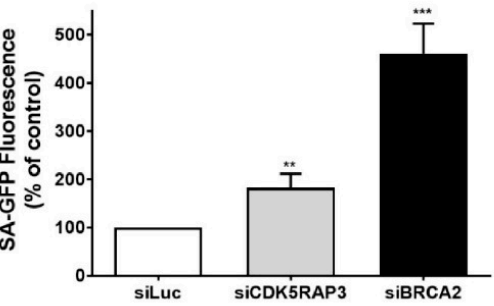

Figure 3. Cont. 
$E$
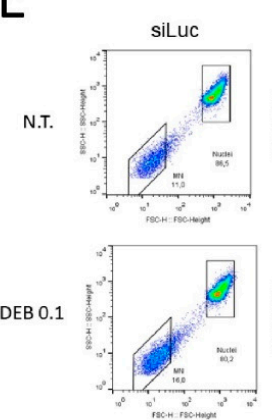
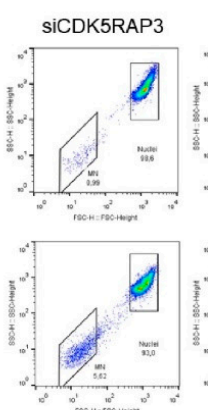
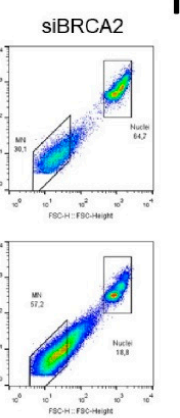

$\mathrm{F}$

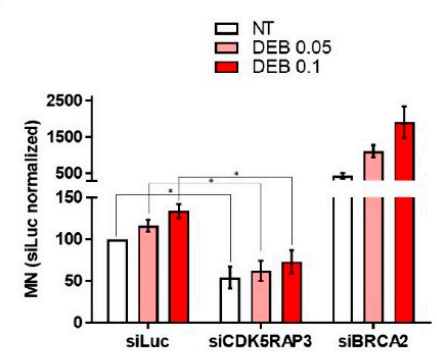

Figure 3. CDK5RAP3 regulates DSB DNA repair and genomic instability by homologous recombination and single-strand annealing. (A) Diagrams showing the in vitro homologous directed and single-strand annealing repair assays (see materials and methods). In the DR-GFP system (upper diagram), U2OS cells carry a plasmid inserted in the genome with two adjacent non-functional GFP coding fragments, one of them with an I-SceI restriction site. When a plasmid expressing I-SceI endonuclease is transfected, a specific DSB is generated in one GFP coding fragment, promoting recombination with the adjacent fragment and the formation of a full, functional GFP coding sequence resulting in fluorescent cells. In the SA-GFP system (lower diagram), U2OS or HeLa cells carry a plasmid inserted in the genome with two adjacent non-functional GFP coding fragments, one short $5^{\prime}$ fragment and a $3^{\prime}$ fragment, including one with an I-SceI restriction site. When a plasmid expressing I-SceI endonuclease is transfected, a specific DSB is generated in the $3^{\prime}$ GFP coding fragment, and a full, functional GFP coding sequence is formed after strand annealing, homolog searching and fusion with the $5^{\prime}$ GFP fragment. (B) Flow cytometry plots from DR-GFP (upper panels) and SA-GFP (bottom panels) in siRNA-transfected U2OS cells. (C) Averaged graph from at least four independent experiments of DR-GFP U2OS cells. GFP-transfected cells were used to normalize transfection efficiency. (D) Averaged graph from at least four independent experiments of SA-GFP in U2OS cells. $(\mathrm{E}, \mathrm{F})$ CDK5RAP3 inhibition reduces spontaneous and DNA damage-induced genomic instability. siRNA-transfected U2OS cells were plated and treated with ICL inducer DEB at different doses, and $72 \mathrm{~h}$ later samples were processed for the FCM assay and analyzed by cell cytometry (see materials and methods and Supplementary Figure S2A). (E) Representative experiment of the FCM assay in U2OS cells. Shown are FACS plots of nuclei and micronuclei (MN). (F) Averaged graph from at least four independent experiments performed as in (E) and normalized to luciferase control samples. Graph bars from $(\mathbf{C}, \mathbf{D}, \mathbf{F})$ show mean \pm SEM. ${ }^{*} p<0.05 * * p<0.01 * * * p 0.001$.

We previously noted in Western blots that BRCA2 expression was consistently increased when CDK5RAP3 was inhibited (Figure 2A, Figures S1A and S5). We wanted to know if other proteins involved in HR repair could have a similar expression profile upon CDK5RAP3 inhibition. As seen in Supplementary Figure S2B,C (and data not shown, uncropped images in Supplementary Figure S5), BRCA2, RAD51 and RPA2 expression increased in CDK5RAP3depleted U2OS and HeLa cells, consistent with a global upregulation of HR repair.

It is well known that the lack of HR repair in BRCA2-deficient cells is overcome by increasing error-prone repair through homology-directed single-strand annealing (SSA) [26]. To determine whether CDK5RAP3 plays a role in SSA, we performed another in vitro cellular assay based on an SSA-dependent correction of an incomplete GFP expression cassette (Figure 3A left panel). Similar to the results of the HR assay, U2OS (Figure 3) and HeLa cells (not shown) with active SSA repair exhibited functional, although less active GFP expression (Figure 3B lower left panel). As anticipated, BRCA2 depletion resulted in a 5- to 30-fold increase in the number of fluorescent cells (Figure 3B lower right panel and 3D). Interestingly, CDK5RAP3 depletion also resulted in a 2- to 10-fold increase in the number of fluorescent cells (Figure 3B lower middle panel and 3D). To further assess the role of CDK5RAP3 in DSB repair, a third GFP reporter-based cellular assay for nonhomologous end joining was performed in U2OS and HeLa cells [27]. This assay showed that depletion of CDK5RAP3 (or BRCA2) had little or no effect on non-homologous end joining (Supplementary Figure S1B-D and data not shown). 
Genomic instability is a hallmark of BRCA2-deficiency upon treatment with chromosomebreaking agents, and frequently micronuclei (MN) production in cells is used to study chromosome fragility [29]. We used the in vitro flow cytometric MN (FCM) assay (see materials and methods) in the presence or absence of CDK5RAP3 [30]. Consistent with DNA damage resistance (Figure 2) and HR and SSA DSB repair induction upon CDK5RAP3 depletion (Figure 3A-D), U2OS and HeLa CDK5RAP3-depleted cells showed a marked reduction in spontaneous and DNA damaging-induced chromosome fragility (Figure 3E,F and data not shown).

\subsection{CDK5RAP3 Expression Is Associated with Poor Breast and Ovarian Cancer Survival}

Given that CDK5RAP3 interacts with BRCA2, whose mutations considerably increase the risk of breast and ovarian cancer, and that CDK5RAP3 downregulation leads to DNA damage resistance, we hypothesized that CDK5RAP3 expression may be associated with poorer survival of breast and ovarian cancer patients. To test this hypothesis, we used the Kaplan-Meyer Plotter cancer transcriptomic database [28]. Data from 3,554 breast cancer patients revealed a significant association between low CDK5RAP3 expression and poor overall survival $\left(\mathrm{HR}=1.61 ; p=3.6 \times 10^{-4}\right)$ and relapse-free survival $(\mathrm{HR}=1.64$; $p<10^{-16}$, Figure 4A,B). A similar but milder association was observed in patients with high-grade serous ovarian cancer (data not shown). Additional analysis also showed an association between poor survival rates and low CDK5RAP3 expression in basal, luminal A and luminal B breast cancer patients (Supplementary Figure S3).

The above data show that lack of CDK5RAP3 leads to increased resistance to DNAdamaging agents in vitro and, in parallel, that low CDK5RAP3 expression is associated with poor breast and ovarian cancer prognosis. To further assess the prediction that low CDK5RAP3 levels promote tumor chemoresistance, we computed the expression correlation between CDK5RAP3 and previously defined signatures of chemoresistance and/or DNA damage resistance using breast cancer data from The Cancer Genome Atlas [31]. The resistance signatures corresponded to sets of genes whose products were involved in epithelial-tomesenchymal transition [32,33], induced by interferon signaling [34], by radiation [35], or associated with poor prognosis triple-negative breast cancer [36]. Remarkably, all analyzed signatures showed a significant negative correlation with CDK5RAP3 (Figure 4B), specially for triple negative breast cancer; that is, low expression of $C D K 5 R A P 3$ was associated with relatively higher expression of the defined signatures, which, in turn, further endorses a functional link between CDK5RAP3 downregulation and chemoresistance.

\subsection{SNPS in CDK5RAP3 May Be Associated with Cancer Risk in BRCA1/2 Mutation Carriers}

Common genetic variation (represented by single nucleotide polymorphisms (SNPs)) at loci encoding for functional interactors of BRCA1 and/or BRCA2 modify breast and/or ovarian cancer risk in carriers of germline $B R C A 1$ and/or $B R C A 2$ mutations $[37,38]$. Since the CDK5RAP3 gene product physically interacts with BRCA2 and functions in the DNA damage response, we conducted an association study using data from the Consortium of Investigators of Modifiers of BRCA1/2, CIMBA [39] (Figure 4C). Combined results of the iCOGS and Oncoarray studies identified potential associations with cancer risk in these settings (minor allele frequency (MAF) $>0.01, p$ values $<10^{-3}$, see supplementary results and methods): BRCA1 mutation carriers, breast cancer risk, imputed rs536194633 $\mathrm{HR}=0.90$; and ovarian cancer risk, genotyped rs2905855 $\mathrm{HR}=1.07$, imputed rs201764131 $\mathrm{HR}=0.91$, and imputed rs6503964 $\mathrm{HR}=0.93 ; B R C A 2$ mutation carriers, breast cancer risk, imputed rs77563750 HR $=1.11$ and rs17617360 $\mathrm{HR}=0.90$. Additional variants showed potential associations with ovarian cancer risk in BRCA2 mutation carriers, but their MAFs were $<0.01$ (imputation $\mathrm{r}^{2}$ values $>0.88$; the complete locus results are shown in Table S1). Bioinformatic analysis of publicly available data suggested an enhancer role on genes at the corresponding genomic region by some of the depicted variants (Supplementary Figure S4, Table S2 and Supplementary Methods). 
A

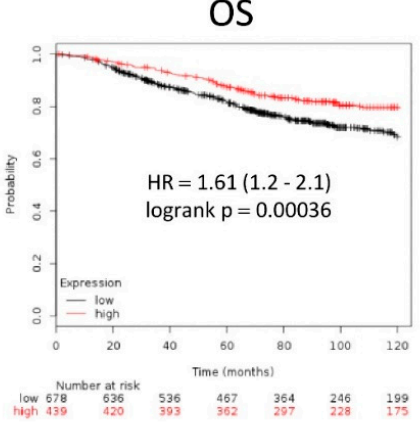

RFS

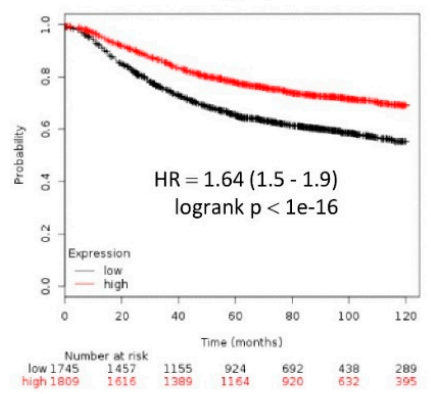

B

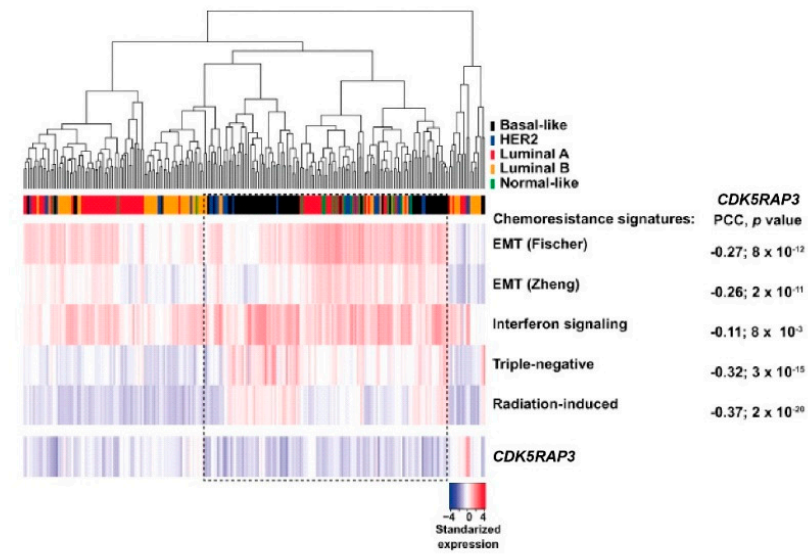

C

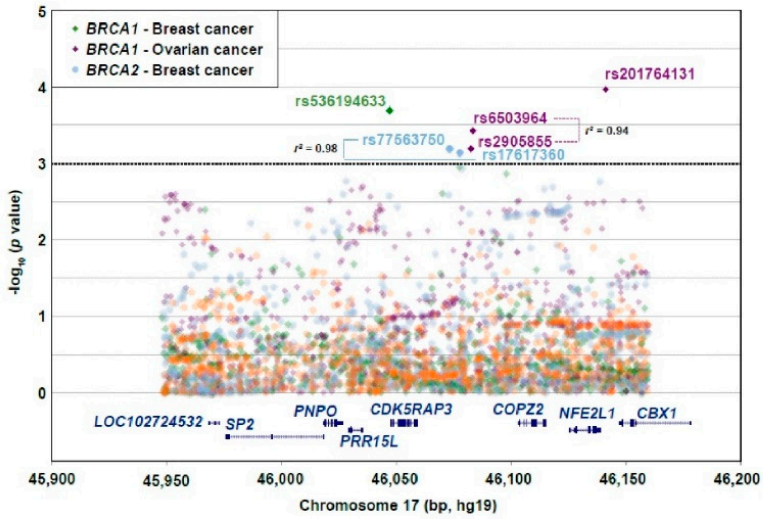

Figure 4. Low $C D K 5 R A P 3$ expression is associated with poor patient survival in breast cancer. (A) Kaplan-Meier survival curves for 1117 (overall survival, OS, left panel) and 3554 (relapse-free survival, RFS) patients with breast cancer. Data were divided at the best cutoff value into high and low expressing groups (for RFS, $n=1745$ for CDK5RAP3 low, $n=1809$ for CDK5RAP3 high; $p<10-16$ with log-rank analysis; for OS, $n=678$ for CDK5RAP3 low, $n=439$ for CDK5RAP3 high; $p=0.00036$ with log-rank analysis). Data obtained from the Kaplan-Meier Plotter (http:/ / www.kmplot.com, accessed on 3 July 2021). (B) Unsupervised clustering of TCGA breast cancer gene expression data of CDK5RAP3 and defined signatures of chemoresistance and/or DNA damage resistance (two sets of epithelial-to-mesenchymal (EMT)-related genes are shown, as indicated in the text). The Pearson correlation coefficients (PCCs), corresponding $p$ values, and tumor subtypes according to PAM50 classification are also shown. The dashed box highlights the cases with relative lower expression of CDK5RAP3 and higher for the signatures. (C) SNPs linked to CDK5RAP3 may influence breast/ovarian cancer risk in BRCA1/2 mutation carriers. Association plot for variants within the genomic region $100 \mathrm{~kb}$ on either side of CDK5RAP3 and risk of ovarian and breast cancer. X-axis position is referent to position (bp) on chromosome 17. Y-axis is - $\log 10$ ( $p$-values) from association tests for risk of breast or ovarian cancer in BRCA1/2 mutation carriers, as shown in the inset. 


\section{Discussion}

Tumor formation and progression result from an imbalance in the expression of oncogenes and tumor suppressors. Oncogenes promote cell growth, and their overexpression or constitutive activation is highly deleterious. In contrast, tumor suppressors are essential for the regulation of uncontrolled cell division and maintenance of homeostasis, and normal cell functions may be compromised when they are downregulated or when their activity is impaired. DNA damage response pathways act as tumor suppressors by maintaining genomic stability and thus preventing oncogenic mutations. Similarly, cell cycle checkpoint pathways also act as tumor suppressors by controlling apoptosis and cell cycle progression, thereby allowing time for DNA repair.

CDK5RAP3 was first suggested to be a tumor suppressor when Wang and co-workers demonstrated functional interactions with $\mathrm{p} 14^{\mathrm{ARF}}$ and $\mathrm{p} 53$ [17]. Shortly thereafter, the same authors reported that CDK5RAP3 inhibits NF- $\mathrm{KB}$ signaling, providing additional evidence for its role in preventing tumor formation. Cells lacking CDK5RAP3 are more prone to cell invasion and exhibit enhanced xenograft tumor growth [18]. Interestingly, one-third of HNSCCs express remarkably low levels of CDK5RAP3, and low CDK5RAP3 levels were associated with reduced survival in HCC patients [20].

In this study, we link for the first time CDK5RAP3 function with DNA repair by demonstrating that it physically interacts with BRCA2 and its absence leads to increased HR and SSA repair pathways. This is in contrast with BRCA2, whose depletion or mutation leads to HR abrogation compensated with induced SSA [26,40,41]. In addition, the observed DNA damage resistance and reduced genomic instability in CDK5RAP3-depleted cells may contribute to increased tumor aggressiveness and progression in breast cancer patients (as seen in Km plot analysis).

By yeast two hybrid experiments, we found that the BRCA2 helical domain interacted with CDK5RAP3 (Figure 1A), and this binding was further validated in co-IP experiments (Figure 1B,C). Some pathogenic missense variants have been identified in BRCA2 that map to the helical domain. They have been classified as pathogenic or not based on its capacity to disrupt HR pathway, but there are no reports on their role in SSA activity [4,42]. Thus, it would be interesting to address their capacity to maintain CDK5RAP3 interaction, in addition to SSA activity. HR induction by lack of CDK5RAP3 may be explained by BRCA2 expression induction (Figure 2A right and Supplementary Figure S2B,C), which could be produced through gene expression regulation at the transcriptional level or through protein stability mechanisms. However, how SSA activity increases in the absence of CDK5RAP3 remains elusive. Further experiments are needed to unravel the mechanisms by which CDK5RAP3 and BRCA2 regulate the HR and SSA pathways.

Our data connecting CDK5RAP3 to the BRCA2 pathway suggest a potential therapeutic approach for breast cancer. Blocking SSA activity is synthetically lethal in BRCA2deficient tumors [43-45]. Similarly, because CDK5RAP3 deficiency could make tumors dependent on HR or SSA repair pathways, it is conceivable that inhibiting HR, SSA or both combined would also be synthetically lethal in tumor cells with low CDK5RAP3 expression. Indeed, HR inhibitors targeting RAD51 and SSA inhibitors targeting RAD52 or XPF have recently been identified and are under development for clinical applications [46-50].

The functional connection between CDK5RAP3 and breast cancer may be extended to cancer etiology, as suggested by the results of the association study between common genetic variants and cancer development in $B R C A 1 / 2$ mutation carriers. The biochemical interaction with BRCA2 and the functional link to the DNA damage response support this hypothesis. In addition, functional annotation of the strongest risk variation indicates potential alterations of regulatory mechanisms. However, further genetic analyses combined with assays measuring the impact of specific variants are warranted in future studies.

In summary, in addition to the link to cancer risk, our study identifies CDK5RAP3 expression in breast tumors as a new biomarker for poor prognosis and potential target for therapeutic intervention. Lack of CDK5RAP3 targets HR and SSA, two crucial tumorsuppressor DSB repair pathways, which can be added to its link with cell cycle arrest and 
p53 [17]. Interestingly, both BRCA2-deficient and p53-deficient mice have a carcinogenic phenotype independently. However, when both genes are simultaneously abrogated, tumor progression is extremely enhanced, and tumor cells acquire resistance to DNA-damaging drugs, a phenotype that resembles our findings related to CDK5RAP3 depletion [51-54]. CDK5RAP3 downregulation may therefore lead to the worst possible scenario for tumor progression because it confers resistance to DNA damage agents, reduces genome instability, and increases tumor aggressiveness.

\section{Conclusions}

CDK5RAP3 is a novel BRCA2-interacting protein that negatively regulates homologous recombination and single-strand annealing. CDK5RAP3 expression is linked to breast cancer survival and its genetic variations are associated with BRCA1/2 mutation carriers. CDK5RAP3 thus emerges as a potential prognostic biomarker for breast cancer.

Supplementary Materials: The following are available online at https: / www.mdpi.com/article/ 10.3390/cancers14020353/s1, Figure S1: CDK5RAP3 regulation in non homologous end joining. Figure S2: Genomic instability by flow cytometric micronucleus (FCM) assay and CDK5RAP3 role in HR-related protein expression. Figure S3: Low CDK5RAP3 expression association with survival curves for different breast cancer subtypes. Figure S4: Functional annotation of the 17q21.32 region showing positions of candidate variants in relation to RefSeq annotated genes. Figure S5: Uncropped Western blots from Figure 2A (A), Supplementary Figure S1A (B) and Figure S2B (C), ladders are shown on the left side of the images, and cropped images marked as blue boxes. Table S1: Results of the combined iCOGS and Oncoarray studies at the CDK5RAP3 locus (imputed and genotyped variants) in BRCA1 and BRCA2 mutation carriers. Table S2: Functional annotation of potentially associated variants at the CDK5RAP3 locus.

Author Contributions: Conception and design: J.M., M.A.P., J.S. Development of methodology: J.M., M.J.R., M.A.P., J.S. Acquisition of data (provided cell lines, acquired and managed patients, provided facilities, etc.): J.M., M.J.R., L.R., P.B.-M., C.C.-F., G.R.d.G., H.S., H.M., R.P., G.H., P.C., P.S., G.M., A.G., D.C., M.J.G., J.G., CIMBA. Analysis and interpretation of data (e.g., statistical analysis, biostatistics, computational analysis): J.M., M.J.R., G.R.d.G., H.S., G.H., M.B., P.C., P.S., G.M., A.G., D.C., M.J.G., J.G., CIMBA, J.B., C.L., F.J.C., M.A.P., J.S. Writing, review, and/or revision of the manuscript: J.M., M.A.P., J.S. Study supervision: J.M., J.S. All authors have read and agreed to the published version of the manuscript.

Funding: Surrallés laboratory is supported by the ICREA Academia programme, Spanish Ministry of Science, Innovation and Universities (projects CB06/07/0023 MCI, UE and RTI2018-098419-B-I00 $\mathrm{MCI} / \mathrm{AEI} / \mathrm{FEDER}, \mathrm{UE}$ ), European Commission (and P-SPHERE COFUND project), Fanconi Anemia Research Fund Inc. This work was also funded by intramural actions (ACCI-U705/U745-2018 to JS) of CIBERER. CIBERER is an initiative of the Instituto de Salud Carlos III, Spain. M.A.P. laboratory was funded by the Instituto de Salud Carlos III grants PI18/01029 and PI21/01306, Generalitat de Catalunya SGR 2017-449, and CERCA Program to IDIBELL. The CIMBA data management and data analysis were supported by Cancer Research UK grants C12292/A20861 and PPRPGM-Nov20 100002. iCOGS: the European Community's Seventh Framework Programme under grant agreement $\mathrm{n}$ 223175 (HEALTH-F2-2009-223175) (COGS), Cancer Research UK (C1287/A10118, C1287 / A 10710, C12292/A11174, C1281/A12014, C5047/A8384, C5047/A15007, C5047/A10692, C8197/A16565), the National Institutes of Health (CA128978) and Post-Cancer GWAS initiative (1U19 CA148537, 1U19 CA148065 and 1U19 CA148112-the GAME-ON initiative), the Department of Defence (W81XWH-10-10341), the Canadian Institutes of Health Research (CIHR) for the CIHR Team in Familial Risks of Breast Cancer (CRN-87521), and the Ministry of Economic Development, Innovation and Export Trade (PSRSIIRI-701), Komen Foundation for the Cure, the Breast Cancer Research Foundation, and the Ovarian Cancer Research Fund. The PERSPECTIVE project was supported by the Government of Canada through Genome Canada and the Canadian Institutes of Health Research, the Ministry of Economy, Science and Innovation through Genome Québec, and The Quebec Breast Cancer Foundation.

Institutional Review Board Statement: Not applicable.

Informed Consent Statement: Not applicable. 
Data Availability Statement: The data presented in this study are available in this article and supplementary materials.

Acknowledgments: The authors want to thank the microscope and cell cytometry facility of the Universitat Autónoma de Barcelona for their help and support.

Conflicts of Interest: The authors declare no conflict of interest. The funders had no role in the design of the study; in the collection, analyses, or interpretation of data; in the writing of the manuscript; or in the decision to publish the results.

\section{References}

1. Ciccia, A.; Elledge, S.J. The DNA Damage Response: Making It Safe to Play with Knives. Mol. Cell. 2010, 40, 179-204.

2. Roy, R.; Chun, J.; Powell, S.N. BRCA1 and BRCA2: Different roles in a common pathway of genome protection. Nat. Rev. Cancer 2012, 12, 68-78.

3. Lee, H. Cycling with BRCA2 from DNA repair to mitosis. Exp. Cell Res. 2014, 329, 78-84. [PubMed]

4. Guidugli, L.; Carreira, A.; Caputo, S.M.; Ehlen, A.; Galli, A.; Monteiro, A.N.A.; Nwuhausen, S.L.; Hansen, T.V.O.; Couch, F.J.; Vreeswijk, M.P.G.; et al. Functional assays for analysis of variants of uncertain significance in BRCA2. Hum. Mutat. 2014, 35, 151-164. [PubMed]

5. Prakash, R.; Zhang, Y.; Feng, W.; Jasin, M. Homologous recombination and human health: The roles of BRCA1, BRCA2, and associated proteins. Cold Spring Harb Perspect Biol. 2015, 7, a016600.

6. Bogliolo, M.; Surrallés, J. Fanconi anemia: A model disease for studies on human genetics and advanced therapeutics. Curr. Opin. Genet. Dev. 2015, 33, 32-40. [PubMed]

7. $\quad$ Easton, D.F.; Lesueur, F.; Decker, B.; Michailidou, K.; Li, J.; Allen, J.; Luccarini, C.; Pooley, K.A.; Shah, M.; Bolla, M.K.; et al. No evidence that protein truncating variants in BRIP1 are associated with breast cancer risk: Implications for gene panel testing. $J$. Med. Genet. 2016, 53, 298-309. [PubMed]

8. Desmond, A.; Kurian, A.W.; Gabree, M.; Mills, M.A.; Anderson, M.J.; Kobayashi, Y.; Horick, N.; Yang, S.; Shannon, K.; Tung, N.; et al. Clinical Actionability of Multigene Panel Testing for Hereditary Breast and Ovarian Cancer Risk Assessment. JAMA Oncol. 2015, 1, 943-951.

9. Knies, K.; Inano, S.; Ramírez, M.J.; Ishiai, M.; Surrallés, J.; Takata, M.; Schindler, D. Biallelic mutations in the ubiquitin ligase RFWD3 cause Fanconi anemia. J. Clin. Investig. 2017, 127, 3013-3027. [PubMed]

10. Walsh, T.; King, M.-C. Ten genes for inherited breast cancer. Cancer Cell 2007, 11, 103-105.

11. Rahman, N.; Seal, S.; Thompson, D.; Kelly, P.; Renwick, A.; Elliott, A.; Reid, S.; Spanova, K.; Barfoot, R.; Chagtai, T.; et al. PALB2, which encodes a BRCA2-interacting protein, is a breast cancer susceptibility gene. Nat. Genet. 2007, 39, 165-167. [PubMed]

12. Southey, M.C.; Fab, K.C.; Teo, Z.L.; Dowty, J.G.; Odefrey, F.A.; Park, D.J.; Tischkowitz, M.; Sabbaghian, N.; Apicella, C.; Byrnes, G.B.; et al. A PALB2 mutation associated with high risk of breast cancer. Breast Cancer Res. 2010, 12, R109.

13. Antoniou, A.C.; Casadei, S.; Heikkinen, T.; Barrowdale, D.; Pylkäs, K.; Roberts, J.; Lee, A.; Subramanian, D.; De Leeneer, K.; Fostira, F.; et al. Breast-cancer risk in families with mutations in PALB2. N. Engl. J. Med. 2014, 371, 497-506. [PubMed]

14. Meindl, A.; Hellebrand, H.; Wiek, C.; Erven, V.; Wappenschmidt, B.; Niederacher, D.; Freund, M.; Lichtner, P.; Hartmann, L.; Schaal, H.; et al. Germline mutations in breast and ovarian cancer pedigrees establish RAD51C as a human cancer susceptibility gene. Nat. Genet. 2010, 42, 410-414.

15. Peterlongo, P.; Catucci, I.; Colombo, M.; Caleca, L.; Mucaki, E.; Bogliolo, M.; Marin, M.; Damiola, F.; Bernard, L.; Pensotti, V.; et al. FANCM c.5791C $>$ T nonsense mutation (rs144567652) induces exon skipping, affects DNA repair activity and is a familial breast cancer risk factor. Hum. Mol. Genet. 2015, 24, 5345-5355.

16. Jiang, H.; Luo, S.; Li, H. Cdk5 activator-binding protein C53 regulates apoptosis induced by genotoxic stress via modulating the G2/M DNA damage checkpoint. J. Biol. Chem. 2005, 280, 20651-20659.

17. Wang, J.; He, X.; Luo, Y.; Yarbrough, W.G. A novel ARF-binding protein (LZAP) alters ARF regulation of HDM2. Biochem. J. 2006, 393, 489-501.

18. Wang, J.; An, H.; Mayo, M.W.; Baldwin, A.S.; Yarbrough, W.G. LZAP, a putative tumor suppressor, selectively inhibits NF-kappaB. Cancer Cell 2007, 12, 239-251. [PubMed]

19. Jiang, H.; Wu, J.; He, C.; Yang, W.; Li, H. Tumor suppressor protein C53 antagonizes checkpoint kinases to promote cyclindependent kinase 1 activation. Cell Res. 2009, 19, 458-468.

20. Zhao, J.-J.; Pan, K.; Li, J.-J.; Chen, Y.-B.; Chen, J.-G.; Lv, L.; Wang, D.-D.; Pan, Q.-Z.; Chen, M.-S.; Xia, J.-C. Identification of LZAP as a New Candidate Tumor Suppressor in Hepatocellular Carcinoma. PLoS ONE. 2011, 6, e26608.

21. Montanuy, H.; Camps-Fajol, C.; Carreras-Puigvert, J.; Haggblad, M.; Lundgren, B.; Aza-Carmona, M.; Helleday, T.; Minguillon, J.; Surralles, J. High content drug screening for Fanconi anemia therapeutics. Orphanet. J. Rare Dis. 2020, 15, 170.

22. Martrat, G.; Maxwell, C.M.; Tominaga, E.; Porta-de-la-Riva, M.; Bonifaci, N.; Gómez-Baldó, L.; Bogliolo, M.; Lazaro, C.; Blanco, I.; Brunet, J.; et al. Exploring the link between MORF4L1 and risk of breast cancer. Breast Cancer Res. 2011, 13, R40.

23. Hernández, G.; Ramírez, M.J.; Minguillón, J.; Quiles, P.; de Garibay, G.R.; Aza-Carmona, M.; Bogliolo, M.; Pujol, R.; Prados, R.; Fernández, J.; et al. Decapping protein EDC4 regulates DNA repair and phenocopies BRCA1. Nat. Commun. 2018, 9, 967. [PubMed] 
24. Montanuy, H.; Martínez-Barriocanal, A.; Casado, J.A.; Rovirosa, L.; Ramírez, M.J.; Nieto, R.; Carrascoso-Rubio, C.; Riera, P.; González, A.; Lerma, E.; et al. Gefitinib and afatinib show potential efficacy for fanconi anemia-related head and neck cancer. Clin. Cancer Res. 2020, 26, 3044-3057.

25. Pierce, A.J.; Johnson, R.D.; Thompson, L.H.; Jasin, M. XRCC3 promotes homology-directed repair of DNA damage in mammalian cells. Genes Dev. 1999, 13, 2633-2638. [PubMed]

26. Stark, J.M.; Pierce, A.J.; Oh, J.; Pastink, A.; Jasin, M. Genetic steps of mammalian homologous repair with distinct mutagenic consequences. Mol. Cell Biol. 2004, 24, 9305-9316.

27. Bennardo, N.; Cheng, A.; Huang, N.; Stark, J.M. Alternative-NHEJ is a mechanistically distinct pathway of mammalian chromosome break repair. PLoS Genet 2008, 4, e1000110.

28. Győrffy, B.; Surowiak, P.; Budczies, J.; Lánczky, A. Online survival analysis software to assess the prognostic value of biomarkers using transcriptomic data in non-small-cell lung cancer. PLoS ONE 2013, 8, e82241.

29. Ramírez, M.J.; Minguillón, J.; Loveless, S.; Lake, K.; Carrasco, E.; Stjepanovic, N.; Balmaña, J.; Català, A.; Mehta, P.A.; Surrallés, J Chromosome fragility in the buccal epithelium in patients with Fanconi anemia. Cancer Lett. 2020, 472, 1-7.

30. Avlasevich, S.L.; Bryce, S.M.; Cairns, S.E.; Dertinger, S.D. In vitro micronucleus scoring by flow cytometry: Differential staining of micronuclei versus apoptotic and necrotic chromatin enhances assay reliability. Environ. Mol. Mutagen. 2006, 47, 56-66. [PubMed]

31. CGAN. Comprehensive molecular portraits of human breast tumours. Nature 2012, 490, 61-70.

32. Fischer, K.R.; Durrans, A.; Lee, S.; Sheng, J.; Li, F.; Wong, S.T.C.; Choi, H.; El Rayes, T.; Ryu, S.; Troeger, J.S.; et al. Epithelialto-mesenchymal transition is not required for lung metastasis but contributes to chemoresistance. Nature 2015, 527, 472-476. [PubMed]

33. Zheng, X.; Carstens, J.L.; Kim, J.; Scheible, M.; Kaye, J.; Sugimoto, H.; Wu, C.-C.; LeBleu, V.S.; Kalluri, R. Epithelial-to-mesenchymal transition is dispensable for metastasis but induces chemoresistance in pancreatic cancer. Nature 2015, 527, 525-530.

34. Weichselbaum, R.R.; Ishwaran, H.; Yoon, T.; Nuyten, D.S.A.; Baker, S.; Khodarev, N.; Su, A.W.; Shaikh, A.Y.; Roach, P.; Kreike, B. et al. An interferon-related gene signature for DNA damage resistance is a predictive marker for chemotherapy and radiation for breast cancer. Proc. Natl. Acad. Sci. USA 2008, 105, 18490-18495. [PubMed]

35. Oh, D.S.; Cheang, M.C.U.; Fan, C.; Perou, C.M. Radiation-induced gene signature predicts pathologic complete response to neoadjuvant chemotherapy in breast cancer patients. Radiat. Res. 2014, 181, 193-207.

36. Liu, Y.-R.; Jiang, Y.-Z.; Xu, X.-E.; Hu, X.; Yu, K.-D.; Shao, Z.-M. Comprehensive Transcriptome Profiling Reveals Multigene Signatures in Triple-Negative Breast Cancer. Clin. Cancer Res. 2016, 22, 1653-1662.

37. Rebbeck, T.R.; Mitra, N.; Domchek, S.M.; Wan, F.; Friebel, T.; Tran, T.V.; Singer, C.F.; Tea, M.-K.M.; Blum, J.L.; Tung, N.; et al. Modification of BRCA1-Associated Breast and Ovarian Cancer Risk by BRCA1-Interacting Genes. Cancer Res. 2011, 71, 5792-5805.

38. Maxwell, C.A.; Benítez, J.; Gómez-Baldó, L.; Osorio, A.; Bonifaci, N.; Fernández-Ramires, R.; Costes, S.; Guinó, E.; Chen, H.; Evans, G.; et al. Interplay between BRCA1 and RHAMM Regulates Epithelial Apicobasal Polarization and May Influence Risk of Breast Cancer. PLoS Biol. 2011, 9, e1001199.

39. Chenevix-Trench, G.; Milne, R.L.; Antoniou, A.C.; Couch, F.J.; Easton, D.F.; Goldgar, D.E.; CIMBA. An international initiative to identify genetic modifiers of cancer risk in BRCA1 and BRCA2 mutation carriers: The Consortium of Investigators of Modifiers of BRCA1 and BRCA2 (CIMBA). Breast Cancer Res. 2007, 9, 104.

40. Tutt, A.; Bertwistle, D.; Valentine, J.; Gabriel, A.; Swift, S.; Ross, G.; Griffin, C.; Thacker, J.; Ashworth, A. Mutation in Brca2 stimulates error-prone homology-directed repair of DNA double-strand breaks occurring between repeated sequences. $E M B O J$. 2001, 20, 4704-4716.

41. Larminat, F.; Germanier, M.; Papouli, E.; Defais, M. Deficiency in BRCA2 leads to increase in non-conservative homologous recombination. Oncogene 2002, 21, 5188-5192.

42. Guidugli, L.; Pankratz, V.S.; Singh, N.; Thompson, J.; Erding, C.A.; Engel, C.; Schmutzler, R.; Domchek, S.; Nathanson, K.; Radice, P.; et al. A classification model for BRCA2 DNA binding domain missense variants based on homology-directed repair activity. Cancer Res. 2013, 73, 265-275.

43. Feng, Z.; Scott, S.P.; Bussen, W.; Sharma, G.G.; Guo, G.; Pandita, T.K.; Powell, S.N. Rad52 inactivation is synthetically lethal with BRCA2 deficiency. Proc. Natl. Acad. Sci. USA 2011, 108, 686-691. [PubMed]

44. Lok, B.H.; Powell, S.N. Molecular pathways: Understanding the role of Rad52 in homologous recombination for therapeutic advancement. Clin. Cancer Res. 2012, 18, 6400-6406.

45. Lok, B.H.; Carley, C.; Tchang, B.; Powell, S.N. RAD52 inactivation is synthetically lethal with deficiencies in BRCA1 and PALB2 in addition to BRCA2 through RAD51-mediated homologous recombination. Oncogene 2013, 32, 3552-3558.

46. Ward, A.; Khanna, K.K.; Wiegmans, A.P. Targeting homologous recombination, new pre-clinical and clinical therapeutic combinations inhibiting RAD51. Cancer Treat Rev. 2015, 41, 35-45. [PubMed]

47. Gavande, N.S.; VanderVere-Carozza, P.S.; Hinshaw, H.D.; Jalal, S.I.; Sears, C.R.; Pawelczak, K.S.; Turchi, J.J. DNA repair targeted therapy: The past or future of cancer treatment? Pharmacol. Ther. 2016, 160, 65-83. [PubMed]

48. Chandramouly, G.; McDevitt, S.; Sullivan, K.; Kent, T.; Luz, A.; Glickman, J.F.; Andrake, M.; Skorski, T.; Pomerantz, R.T. Small-Molecule Disruption of RAD52 Rings as a Mechanism for Precision Medicine in BRCA-Deficient Cancers. Chem. Biol. 2015, 22, 1491-1504.

49. Huang, F.; Goyal, N.; Sullivan, K.; Hanamshet, K.; Patel, M.; Mazina, O.M.; Wang, C.X.; An, W.F.; Spoonamore, J.; Metkar, S.; et al. Targeting BRCA1- and BRCA2-deficient cells with RAD52 small molecule inhibitors. Nucleic Acids Res. 2016, 44, 4189-4199. 
50. Sullivan, K.; Cramer-Morales, K.; McElroy, D.L.; Ostrov, D.A.; Haas, K.; Childers, W.; Hromas, R.; Skorski, T. Identification of a Small Molecule Inhibitor of RAD52 by Structure-Based Selection. PLoS ONE 2016, 11, e0147230.

51. Jonkers, J.; Meuwissen, R.; van der Gulden, H.; Peterse, H.; van der Valk, M.; Berns, A. Synergistic tumor suppressor activity of BRCA2 and p53 in a conditional mouse model for breast cancer. Nat. Genet. 2001, 29, 418-425.

52. Jonkers, J.; Berns, A. Conditional mouse models of sporadic cancer. Nat. Rev. Cancer 2002, 2, 251-265. [PubMed]

53. Holstege, H.; Joosse, S.A.; van Oostrom, C.T.M.; Nederlof, P.M.; de Vries, A.; Jonkers, J. High incidence of protein-truncating TP53 mutations in BRCA1-related breast cancer. Cancer Res. 2009, 69, 3625-3633. [PubMed]

54. Bowman-Colin, C.; Xia, B.; Bunting, S.; Klijn, C.; Drost, R.; Bouwman, P.; Fineman, L.; Chen, X.; Culhane, A.; Cai, H.; et al. Palb2 synergizes with Trp53 to suppress mammary tumor formation in a model of inherited breast cancer. Proc. Natl. Acad Sci. USA 2013, 110, 8632-8637. [PubMed] 